\title{
Monte Carlo simulation of kinetically slowed down phase separation $^{\star}$
}

\author{
Štěpán Rưžička ${ }^{1,2, a}$ and Michael P. Allen ${ }^{2,3, b}$ \\ 1 Laboratoire de Physique des Solides, Université Paris-Sud \& CNRS, UMR 8502, 91405 Orsay, France \\ 2 Department of Physics, University of Warwick, Coventry CV4 7AL, UK \\ ${ }^{3}$ H. H. Wills Physics Laboratory, Royal Fort, Tyndall Avenue, Bristol BS8 1TL, UK
}

Received 8 April 2015 and Received in final form 24 May 2015

Published online: 30 June 2015

(C) The Author(s) 2015. This article is published with open access at Springerlink.com

\begin{abstract}
Supercooled colloidal or molecular systems at low densities are known to form liquid, crystalline or glassy drops, which may remain isolated for a long time before they aggregate. This paper analyses the properties of this large time window, and how it can be tackled by computer simulation. We use singleparticle and virtual move Monte Carlo simulations of short-range attractive spheres which are undercooled to the temperature region, where the spinodal intersects the attractive glass line. We study two different systems and we report the following kinetic behavior. A low-density system is shown to exhibit universal linear growth regimes under single-particle Monte Carlo correlating the growth rate to the local structure. These regimes are suppressed under collective motion, where droplets aggregate into a single large disordered domain. It is shown that the aggregation can be avoided and linear regimes recovered, if long-range repulsion is added to the short-range attraction. The results provide an insight into the behavior of the virtual move algorithm generating cluster moves according to the local forcefields. We show that different choices of maximum Monte Carlo displacement affect the dynamical trajectories but lead to the same kinetically slowed down or arrested states.
\end{abstract}

\section{Introduction}

Phase separation of undercooled systems at low densities is a common mechanism of colloidal, molecular or atomistic crystallization. An example is the controlled fabrication of nanocrystals, and their self-assembly into larger structures known as colloidal crystals or crystals formed by oriented attachment or mesocrystallization $[1,2]$. The crystallization in those systems may be initiated by a phase separation into locally dense regions (drops) [3$6]$, which may remain isolated for a very long time $[7,8]$. These drops are typically metastable and may be in a liquid, crystalline, or glassy [9] state before they aggregate or dissolve. The properties of the state depend on the interplay between various parameters of the system such as the temperature, strength of the attraction [10], timescale [9], or viscosity of the solvent affecting the diffusion rate [11, 5]. For example, crystallization within the drops may precede the aggregation, if the temperature of the quench lies

\footnotetext{
* Supplementary material in the form of a .pdf file available from the Journal web page at

http://dx.doi.org/10.1140/epje/i2015-15068-5

a e-mail: stepan.ruzicka@u-psud.fr

b e-mail: m.p.allen@bristol.ac.uk
}

above the intersection of the spinodal with the extrapolated glass line $[8,12,13]$. If the temperature is below this intersection, the drops may remain in the form of pieces of attractive glass $[9,8]$, where the crystallization is logarithmically slowed down (not completely arrested [14]). These glassy regions eventually aggregate into a gel-like structure [15].

Computer simulation is a commonly used tool to study the phase separation [8,3,16-19], with standard molecular dynamics (MD) or Brownian dynamics methods being the main simulation approaches. These methods provide a good insight into shorter timescales at which the initial fluid condenses into the drops, and at which the crystallinity within the drops evolves. However, the timescale needed for large scale motion or aggregation of the drops is long and remains typically unexplored [8]. Moreover, as described by the Stokes law of hydrodynamics for spherical objects in a fluid [20], the diffusion rates of single particles, small clusters, and large clusters, cover a wide range, which limits the efficiency of standard, realistic, simulation methods. Accordingly, there is some interest in applying artificially accelerated simulation methods, together with an attempt at mapping the observed kinetics back onto physically realistic timescales. 


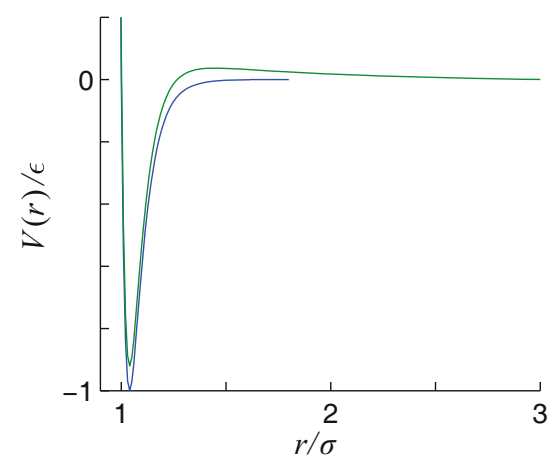

Fig. 1. (Color online) Short-range attractive 36-18 $(\alpha=18)$ Lennard-Jones potential with (green, SALR) and without (blue, SA) long-range Yukawa repulsion.

In this paper, we study a standard simulation model for colloidal gelation $[3,21,8]$ : short-range attractive (SA) spheres with a repulsive core and with the range of attraction being approximately $20 \%$ of the core diameter (fig. 1). The phase diagram of this model is well explored [3]; the metastable gas-liquid binodal is buried below the fluid-solid coexistence line. This system is well known to undergo strong clustering into metastable drops after quenching to low densities $[3,8]$. In an attempt to explore those parts of phase space which are hardly accessible to an MD-based algorithm, we use an enhanced Monte Carlo (MC) technique similar to that of configurationalbias Monte Carlo [22]. Since the long-living kinetically slowed down phase studied in this paper is a direct consequence of kinetics (competing with thermodynamics [18]), we use kinetic, or local, MC moves [23]. This is in contrast to non-local moves $[24,25]$, which mix the states only in order to efficiently sample thermodynamic states of the system without considering the effect of the kinetics. If non-local moves were used [19], the system studied here would eventually reach the thermodynamically stable state where gas coexists with a crystal. Translational and rotational MC cluster moves [20,18,26,27], known as virtual move Monte Carlo (VMMC), have been used here to model the dynamics. The algorithm selects the moving particles or clusters according to the local forcefields, and turned out to be particularly efficient in the simulation of aggregation in low-density systems [18,26,27]. Another advantage of the VMMC algorithm is the ability to control the acceptance rate of differently sized moving clusters, thus distinguishing between their diffusion rates.

The simulation models and methods are described in sect. 2. In sect. 3, we examine the differences between the phase separation trajectories of the SA system both under single-particle Monte Carlo (sect. 3.1) and VMMC (sect. 3.2) motion. We show that, if collective motion is forbidden, phase-separating drops grow via three linear growth regimes, and crystallization within the drops precedes the aggregation. To investigate the effect of charge or steric [28] stabilization, in sect. 3.3 we study the trajectories of a system that is identical, except that the SA particles also repel each other at long distances, the so-called short-range attractive and long-range repulsive
(SALR) interaction. The long-range repulsion may also represent accumulation [29] repulsion (see Supplementary Information (SI)). It is shown that the long-range repulsion stabilizes the drops against aggregation, and that, similarly to single-particle Monte Carlo, the growth proceeds via single-particle exchange characterized by linear growth regimes. In sect. 4 , we question whether the MC dynamics is independent of the maximum MC displacement $\delta$. We show that the initial dynamics scales reasonably well with $\delta$, provided the growth proceeds mainly via single-particle exchange. However, if collective motion is allowed, the phase separation trajectories are entirely different for different choices of $\delta$. In sect. 5 , we discuss how to optimally choose and interpret the maximum displacement $\delta$. We conclude in sect. 6 .

\section{Methods}

Particles in our system attract each other via a shortrange attraction $[3,8]$, typically representing colloidal depletion or Van der Waals interactions. Particles may also repel each other at long distances via a screened electrostatic repulsion $[30,21,31,32]$. Systems of these particles are quenched from the fluid phase to the region of the phase diagram where condensation into liquid drops ensues. Monte Carlo techniques [18,26,27] are then used to study the competition between the kinetics of crystallization in the drops, and the aggregation of the drops into a single domain, or multiple metastable domains.

\subsection{Simulation model}

We use a system of $N=2000$ particles interacting via the potential

$$
V(r)=A \frac{e^{-r / \xi}}{r / \xi}+4 \epsilon\left[\left(\frac{\sigma}{r}\right)^{2 \alpha}-\left(\frac{\sigma}{r}\right)^{\alpha}\right]-V_{c},
$$

where the first term is the long-range Yukawa repulsion, and the second term is the generalized Lennard-Jones (LJ) short-range attraction [3]. The parameter $A$ is the Yukawa amplitude, $\xi$ is the screening length, $\sigma$ represents the diameter of the repulsive core, $\epsilon$ is the strength of the shortrange attraction, and $\alpha$ controls the attraction width. $V_{c}$ is chosen such that $V\left(r_{c}\right)=0$. We took $\alpha=18, \sigma=1.0$, $\epsilon=1.0$. To simulate the SA model we fix $A=0.0, r_{c}=1.8$; to simulate the SALR model we take $A=0.08, \xi=2.0$, $r_{c}=3.0$. These potentials are plotted in fig. 1 , and are similar to those of ref. [32], except that the repulsion is weaker.

The simulations were carried out in the constant- $N V T$ ensemble, with low packing fraction $\phi=\pi / 6 \cdot N / V=$ $0.01[8,33]$. The system is quenched from a high temperature fluid to $T=0.25$, which seems to be close to the region of the kinetic phase diagram [15], where the spinodal intersects the extrapolated glass line, and where phase separation starts to be logarithmically slowed down to an 
extent that long-lived disordered aggregates can be observed [8]. Moreover, this temperature lies on the boundary of the optimum region for self-assembly of a similar short-range attractive colloidal system, gleaned from analyzing the fluctuation-dissipation ratios [34,35]. We thus expect both the large-scale aggregation of drops, and the evolution of local order, to happen simultaneously on the timescales we shall consider. To study directly how longrange repulsion between particles affects the kinetics of phase separation, the same temperature is used for the short-range attractive system with long-range repulsion. The inverse temperature is denoted as $\beta=1 / k_{B} T$, where $k_{B}$ is the Boltzmann constant.

The simulations of kinetic arrests (or more precisely kinetically slowed down phase separations $[36,8])$ start from a randomly distributed set of particles without high energetic overlaps. Since we start from a fluid, but under conditions corresponding to an equilibrium state in which the crystal coexists with the gas, and since no time is given to the system to equilibrate at intermediate conditions between these two states, our simulation protocol is referred to as a rapid (non-equilibrium or instantaneous) quench, as opposed to a slow (equilibrium or finite) quenching [9, 37,38 , where the system is allowed at least a short time to find a local equilibrium, and locally phase-separate.

\subsection{Simulation methods}

We use the standard single-particle Monte Carlo (SPMC) algorithm [39], and the symmetrized version [18] of the VMMC algorithm [20], which was formally described and extended by us in refs. $[26,27]$. A MC sweep in the SPMC simulation consists of $N$ single-particle translational moves. In the VMMC simulation, it consists of $N$ virtual translational or rotational moves, selected randomly with $50 \%$ probability. For simplicity, we describe here only the translational moves; the rotational moves were performed in the way described in ref. [26].

The main features of the algorithm can be summarized as follows. Let us consider two states $\mu$ and $\nu$ of our system, which will differ by the position of a group of particles called the moving cluster $\mathcal{C}$. The difference is a small (rigid) translation applied to all particles in $\mathcal{C}$. The number of particles in $\mathcal{C}$ will be denoted as $n_{\mathcal{C}}$. We shall consider different particle pairs $(i, j), i \neq j$. Position vectors of $i$ and $j$ are denoted as $\boldsymbol{r}_{i}$ and $\boldsymbol{r}_{j}$. $M$ is the MC map, meaning that position vectors of $i$ after applying $M$ are denoted as $\boldsymbol{M} \boldsymbol{r}_{i}$. We will consider two relative separations of particles in pair $(i, j)$ : separation (in the original state $\mu$ ) given by $\left(\boldsymbol{r}_{i}, \boldsymbol{r}_{j}\right)$; and separation (in a so-called virtual state $\left.\mu_{i}\right)$ given by $\left(M \boldsymbol{r}_{i}, \boldsymbol{r}_{j}\right)$. The respective pairwise energies are denoted as $\epsilon_{i j}^{(\mu)}, \epsilon_{i^{\prime} j}^{(\mu)}$. The collective MC move can then be summarized as

1. Pick a random particle, and use it as the first (root) particle of the cluster $\mathcal{C}$. Pick a random unit vector $\boldsymbol{u}$, determining the orientation of translation. Pick a random number $a$ drawn uniformly from $(-\delta, \delta)$, determining the magnitude of translation. The position vector of particle $i$ after applying move map $M$ can be expressed as $M \boldsymbol{r}_{i}=\boldsymbol{r}_{i}+a \cdot \boldsymbol{u}$.

2. Pick a random number $b \in \mathcal{U}(0,1)$, and define the maximum number of particles in the cluster as $N_{\mathcal{C}}=$ $1 / b$. (This limit aims to mimic freely draining diffusion of differently sized clusters [20].)

3. Perform a recursive loop selecting all other particles not in $\mathcal{C}$.

(a) Pick randomly a pair $(i, j), i \in \mathcal{C}, j \notin \mathcal{C}$, which interacts in $\left(\boldsymbol{r}_{i}, \boldsymbol{r}_{j}\right)$ or in $\left(M \boldsymbol{r}_{i}, \boldsymbol{r}_{j}\right)$, and to which a link has not yet been proposed. If no such pair exists, finish the cluster selection by exiting the recursive loop.

(b) Test for reaching the maximum number of particles in the cluster: If $n_{\mathcal{C}}>N_{\mathcal{C}}$, label $(i, j)$ as forced failed, and go to (a). Carry on, otherwise.

(c) Attempt to create a link between $(i, j)$ as follows. i. Form a pre-link with probability

$$
p_{i^{\prime} j}^{(\mu)}=\max \left\{0,1-\exp \left(-\beta\left(\epsilon_{i^{\prime} j}^{(\mu)}-\epsilon_{i j}^{(\mu)}\right)\right)\right\} .
$$

ii. If the pre-link does not form, label $(i, j)$ as outright failed, go to (a).

iii. If the pre-link forms, calculate the reverse link formation probability

$$
p_{i j^{\prime}}^{(\mu)}=\max \left\{0,1-\exp \left(-\beta\left(\epsilon_{i j^{\prime}}^{(\mu)}-\epsilon_{i j}^{(\mu)}\right)\right)\right\},
$$

where $\epsilon_{i j^{\prime}}^{(\mu)}$ denotes the energy of $(i, j)$ in $\mu$ after applying the move map $M$ only to $j$, and form the link with probability

$$
\min \left\{1, p_{i j^{\prime}}^{(\mu)} / p_{i^{\prime} j}^{(\mu)}\right\} .
$$

(d) If the link does not form, label $(i, j)$ as frustrated, go to (a).

(e) If the link forms, include $j$ into $\mathcal{C}$, go to (a).

4. Identify the boundary $\mathcal{B}$ of cluster $\mathcal{C}$ as those pairs $(i, j), i \in \mathcal{C}, j \notin \mathcal{C}$, to which a link was proposed but failed to form, i.e. is either outright failed, forced failed, or frustrated.

5. Divide $\mathcal{B}$ into forced failed pairs $\mathcal{B}^{\dagger}$, and all other pairs $\mathcal{B}^{*}$.

6. Accept the cluster move with probability

$$
W_{\text {acc }}^{(\mu \rightarrow \nu \mid R)}=\min \left\{1, \prod_{(i, j) \in \mathcal{B}^{\dagger}} \exp \left(-\beta\left(\epsilon_{i^{\prime} j}^{(\mu)}-\epsilon_{i j}^{(\mu)}\right)\right)\right\},
$$

provided $\mathcal{B}^{*}$ only contains outright failed links. If $\mathcal{B}^{*}$ contains a frustrated link, the move of $\mathcal{C}$ is rejected.

Isotropic ordering [26] of the cluster is used across all our VMMC simulations. The role of energy gradient in selecting the cluster can be alternatively seen from another form of the linking function described in the SI and in ref. [26].

In the following, the physical time $\tau$ is defined as

$$
\tau=n_{\text {sweep }} \cdot \delta^{2},
$$


where $n_{\text {sweep }}$ is the number of MC sweeps, and $\delta$ is the displacement parameter. The time in eq. (3) arises from identifying a diffusion coefficient $D$ from a single-particle random walk in three dimensions

$$
\left\langle\left|\boldsymbol{r}_{i}(\tau)-\boldsymbol{r}_{i}(0)\right|^{2}\right\rangle=n_{\text {sweep }} \delta^{2}=\frac{\tau}{\Delta \tau} \delta^{2}=6 D \tau,
$$

where we associate the time interval $\Delta \tau$ with each Monte Carlo sweep. In SPMC, this corresponds to one attempted move per particle. Under the assumption that the simulation behaves like a diffusive process [40], and not accounting for the effect of rejecting MC trial moves, our choice corresponds to (arbitrarily) fixing the physical "diffusion coefficient" such that $6 D=1$, and $\Delta \tau=\delta^{2}$, in the reduced units defined by the interaction potential. These assumptions and approximations are not correct, since the acceptance probability is lower than one and changes in time during the phase separation process. Moreover, the time step associated with a translational cluster move is proportional to the cluster size according to the Stokes equation as $\tau \propto R_{t} \delta_{t}^{2}$, where $R_{t}$ is the hydrodynamic radius of the cluster corresponding to translation with a certain direction and magnitude $\delta_{t}$. A similar expression for rotations reads $\tau \propto R_{r}^{3} \delta_{r}^{2}$. These relations are derived in ref. [41], are constant across the simulation. It is shown in the SI that this assumption is not valid in our simulations, and that the acceptance ratio changes dramatically in time. An integration based on the local acceptance rate would be needed to approximate the time step. This is outside the scope of the present paper. By plotting the observables as functions of $\tau$ defined by eq. (4) even for VMMC, we highlight the deviations from simple diffusive scaling. We expect these deviations to grow with $\delta$.

\subsection{Analysis}

To analyze the speed of aggregation and stability of cluster phases, we monitor the size of phase separated aggregates. We say that particles $i$ and $j$ are neighbors, denoted $(i, j)$, if they are separated by a distance less than $1.4 \sigma$. This distance roughly corresponds to the local maximum in the interaction potential in eq. (1), but is more universal, and can characterize clustering in systems of attractive particles with a repulsive core, or even just hard spheres [42]. A cluster (also domain or aggregate) is a group of particles which can be connected through a set of neighboring particles, i.e. particles $k$ and $l$ are members of the same cluster if and only if there is a set of neighbors such that $(k, a),(a, b), \ldots,(y, x),(x, l)$. The number of particles within a cluster is defined as the cluster size. It must be stressed that the cluster is generally different from what we term the moving cluster $\mathcal{C}$. The moving cluster is the set of particles that are moved within the VMMC cluster move. The moving cluster is generated randomly, and although it often spans the entire cluster which contains the root particle, it may only be a subset of the cluster, or it may extend across different clusters. We will typically monitor the time evolution of the largest few clusters. In the SI we discuss how the distribution of moving clusters evolves in time.

The time evolution of local crystallinity will be analyzed with $q_{6} q_{6}$ "Steinhardt" parameters $[43,44,42]$. If $N_{b}(i)$ is the number of neighbors of particle $i$, the orientational structure can be characterized by

$$
\begin{aligned}
Q_{l m}(i) & =\frac{1}{N_{b}(i)} \sum_{j=1}^{N_{b}(i)} Y_{l m}\left(\boldsymbol{r}_{i j}\right) \\
q_{l m}(i) & =\frac{Q_{l m}(i)}{\left(\sum_{m^{\prime}=-l}^{l}\left|Q_{l m^{\prime}}(i)\right|^{2}\right)^{1 / 2}}
\end{aligned}
$$

where $Y_{l m}\left(\boldsymbol{r}_{i j}\right)$ is the spherical harmonic corresponding to the orientation vector $\boldsymbol{r}_{i j}$ pointing from particle $i$ to particle $j$. It is known that the crystal structure of the $36-18$ LJ system developed after a suitable quench is a mixture of hexagonal and face-centered cubic lattices, with a slight predominance of the hexagonal structure [8]. We thus focus on $l=6$. A neighboring pair $(i, j)$ is said to be orientationally bonded if

$$
\sum_{m=-6}^{6} q_{6 m}(i) \bar{q}_{6 m}(j)>0.7
$$

where $\bar{q}_{6 m}(j)$ is the complex conjugate of $q_{6 m}(j)$. The number of neighbors which are orientationally bonded to $i$ is denoted as $n_{b}(i)$. A particle $i$ is called crystalline if $n_{b}(i)=12$, and weakly bonded if $n_{b}(i)=2$. A group of at least two bonded crystalline particles, will be called a crystal. A group of at least two particles with $n_{b}(i)>5$, will be called a Low Symmetry Cluster (LSC). In analogy to ref. [42], we will be distinguishing between the LSC with and without a crystal inside.

To characterize the products of a kinetically slowed down phase separation we will define the disordered aggregate as a low-density cluster, which has an aspherical and concave geometry. We typically assume that the aggregate does not disintegrate over a sufficiently long trajectory, and that it is a product of a kinetically slowed down phase separation $[8,36,14]$. It will be seen that these assumptions are satisfied from monitoring the structure of clusters and from the behavior of the VMMC. The definition of a disordered aggregate is different from the definition of a gel $[37,21]$ in that the system does not need to percolate through the periodic boundary conditions in at least $50 \%$ of randomly chosen conformations.

\section{Dynamics of undercooled systems}

In what follows, a low-density short-range attractive fluid is quenched to the temperature $T=0.25$. An SPMC simulation is firstly used to study the limiting dynamics where single particles and small clusters diffuse easily compared to large clusters which move very slowly only as a result of single-particle movements within the cluster. The role of collective motion is then studied by VMMC, which 
considers local forcefields to select the moves of both single particles and clusters. Similarly to what has been reported before [18], it is shown that the collective motion leads to aggregation of the drops into a gel-like structure. Following on from this, we study the time evolution of local crystallinity, and how it competes with aggregation, if long-range repulsion is added to the attraction.

\subsection{Coarsening in attractive systems}

Let us firstly investigate coarsening under SPMC motion in the SA system of attractive spheres $(A=0$ in eq. (1)), quenched to a temperature $T=0.25$. The dynamics of this model can represent a realistic physical system, where single particles diffuse significantly faster than large clusters. The following analysis allows us to compare with collective motion, and to spotlight the coarsening mechanisms in the limiting case where only single particles are allowed to move. The self-assembly and coarsening phenomena in more physically realistic systems can then be understood as a superposition of processes and growth regimes which are consequences of either collective or single-particle motion.

Figure 2 shows that the particles condense into several, long-lived domains, and that the phase separation can be characterized by three different growth regimes, with distinct growth rates.

The first stage of coarsening is characterized by a time window with a large number of clusters (fig. 2(a)) and with the dominant number of particles having a low number of orientational bonds (fig. 2(b)). (See also SI for more details.) The high number of clusters implies a large surface area, and hence low thermodynamic stability and low repulsive (kinetic) barrier to detachment. Consequently, the reorganization is fast: particles easily detach, diffuse, and attach $[45,35]$. The time window is characterized by a fast and linear increase in the number of particles in crystals and in LSCs of both kinds. The total number of crystals and LSCs of both kinds increases rapidly and reaches a maximum, signaling the end of this time window (compare also with fig. 2(c)). The end is also characterized by approximately equal concentrations of weakly bonded and crystalline particles (fig. 2(b)). Figure 2(c) implies that LSCs can contain more than one crystal, and that new crystals generally nucleate only during this time window, and not at later stages of the simulation.

The second stage is dominated by dissolution of disordered clusters. Figure 2(b) shows that the concentration of weakly bonded particles is gradually exceeded by more strongly bonded particles. Growth of the largest domains is slower than in the previous regime, because the number of clusters (and hence the total surface area) is decreasing, and particles are attached to their clusters by an increasing number of bonds. Nevertheless, the growth is faster than in the upcoming time window, because the largest domains grow not only by adsorption of smaller crystals, but mainly because of the presence of LSCs without crystals or even of less well-ordered clusters. Indeed, fig. 2(c) shows that the number of LSCs without crystal
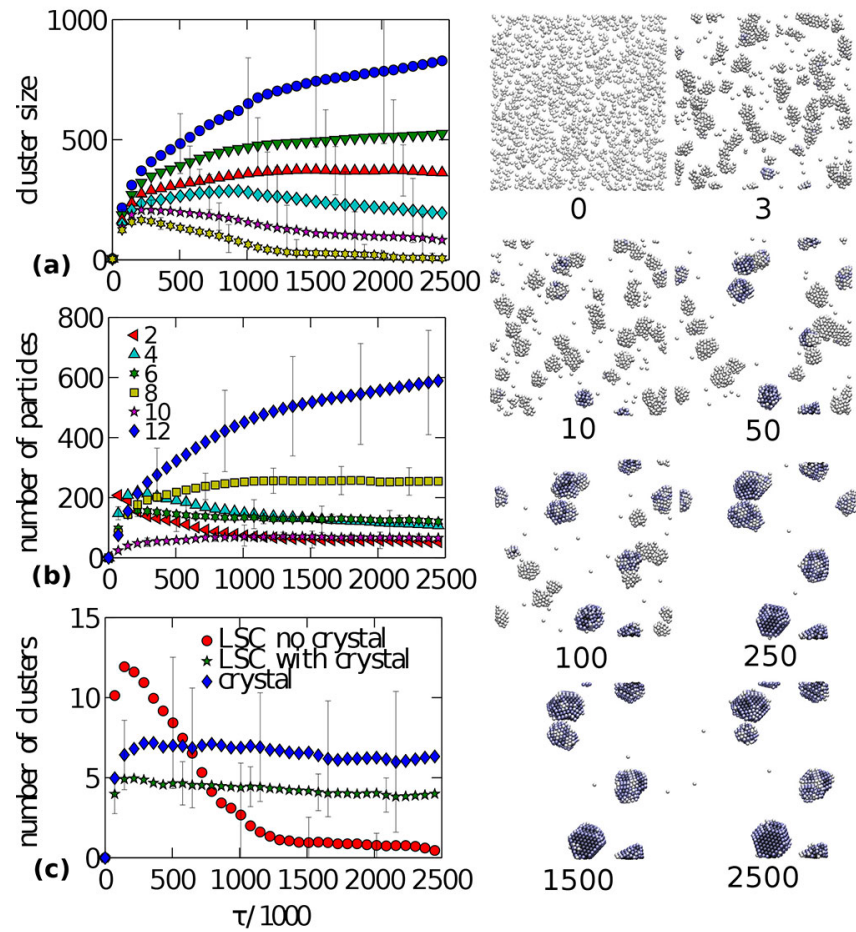

Fig. 2. (Color online) Time evolution of the system under single-particle motion. Here, and elsewhere, time is measured in terms of the quantity $\tau$ defined by eq. (3). The particles are unlikely to escape the crystalline droplets which themselves hardly move. Maximum MC displacement is $\delta=0.30 \sigma$. Particles in the snapshots are colored according to the number of orientational bonds $n_{b}$ ascendingly from white via blue to dark grey. (a) Size of the first six largest clusters. (b) Local order measured by the number of particles with a fixed number of orientational bonds given in the legend. (c) Number of crystals (diamonds), low-symmetry clusters (stars), and low-symmetry clusters with crystals inside (circles). All error bars are estimated from ten independent simulations.

cores undergoes a rapid and distinct period of decrease, and that most clusters which survive this window have a crystal core inside. Figure 2(b) then implies that crystals within LSCs grow during this intermediate time window. The window exists because the timescale needed for crystallization is much longer than the timescale needed to dissolve the low-structured clusters.

The final stage is a growth region with a low concentration of weakly bonded particles. The averages in fig. 2(c) show that the system has several growing and several dissolving clusters. The number of particles with six and eight orientational bonds does not change, indicating that the crystalline surfaces and edges of the domains are well developed. The average number of particles in LSCs is also approximately constant, pointing to the fact that large clusters grow by adsorbing the smaller ones. The growth rate is again smaller than in the previous window, because the overall surface area of the clusters is smaller and because, to detach a particle from a well-formed surface, more bonds need to be broken than to detach a particle 

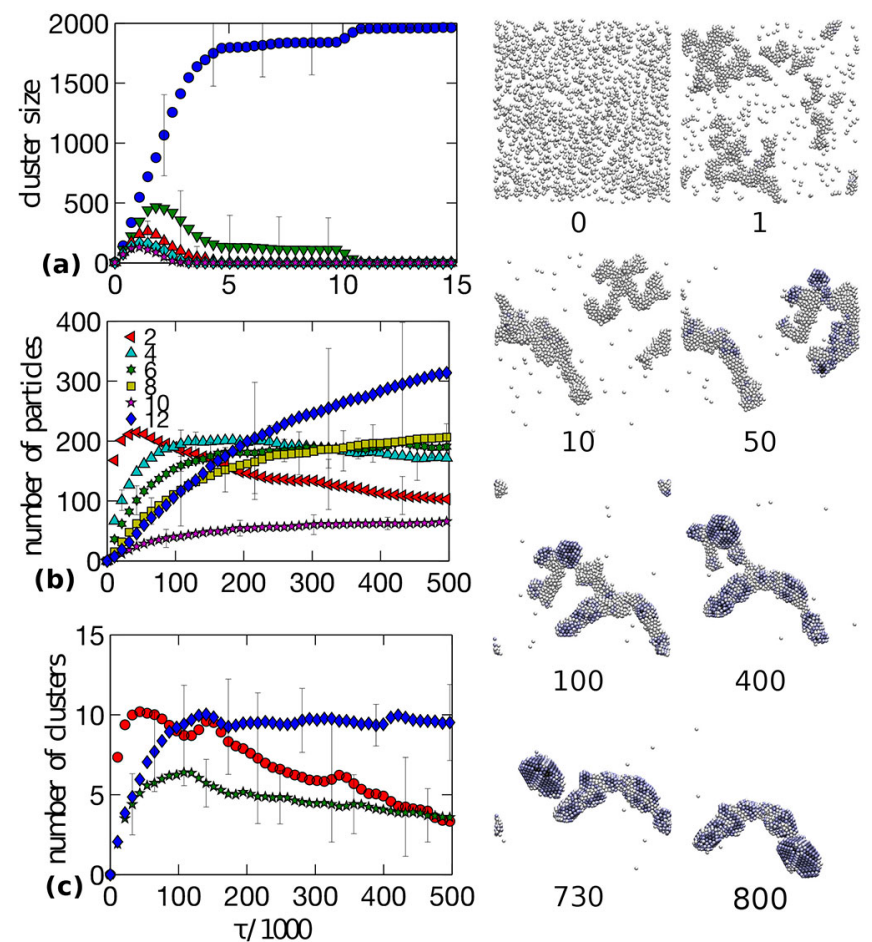

Fig. 3. (Color online) The time evolution of a system where collective motion is allowed leads to the rapid formation of amorphous droplets, which coalesce into a disordered aggregate. Maximum MC displacement is $\delta=0.30 \sigma$. Crystals within this aggregate develop later and are separated by a disordered (liquid) surface. Figures and symbols have meaning analogous of that in fig. 2 .

from a LSC or from the disordered (liquid) surface of a crystal. The growth rate of the largest domain is linear on the timescale of our simulation window, and crystals within LSCs keep growing linearly but at a slower pace than in the previous window (fig. 2(c)).

A comparison of the linear growth stages with the previous analytical [5] or simulation [8] studies would be an interesting topic but has not been done here.

\subsection{Aggregation in attractive systems}

We now consider the same system as in the previous section, except that VMMC is used instead of SPMC. Figure 3 shows that particles merge very quickly into a single cluster. Similarly to SPMC, the first stage of the simulation leads to a rapid clustering within the system into a large number of small clusters. Contrary to SPMC, small clusters not only coarsen but also move easily, and the growth proceeds not only via single-particle exchange, but also via cluster-cluster attachment. This is because the timescale for detachment of a single particle from its cluster and its diffusion to another cluster is comparable to the timescale needed for the whole cluster to diffuse that distance. The locus of states at which this occurs was defined as "clustodal" (by analogy with binodal and spinodal) in ref. [18]. Since small clusters diffuse faster than large clusters, we can generally say that large clusters grow by adsorbing the smaller ones. The aggregation mechanism is so fast that the correlation between the short- and long-range structure known from SPMC is now entirely suppressed.

After a very short time, the second largest cluster reaches its peak size, and starts dissolving, at the expense of the largest one. The dissolution rate is significantly faster than in the SPMC. Figure 3(b) shows that this is caused by a small number of bonds with which a particle is attached to its cluster. The collective motion, allowing whole groups of particles to detach from their clusters and diffuse, and a low repulsive kinetic barrier [46] in locally dense disordered regions, may also accelerate the dissolution process. The dissolution then, on average, stops, and the cluster size reaches a plateau before the second largest cluster dissolves or merges with the largest cluster. Large clusters generally survive longest, because of slow diffusion, and because they are more likely to contain a crystal or a LSC which take longer to dissolve.

Figures 3(a) and 3(c) show that after some time $\left(50 \cdot 10^{3}\right.$ VMMC sweeps), there are on average only two clusters and more than six LSCs, and their number is growing even if the number of clusters remains the same. Figure 3(b) shows that this initial time window is dominated by particles embedded in aggregates with weakly bonded particles. Single-particle rearrangements within these aggregates are easy and local order grows quickly, because the moving clusters are selected through the local energy gradients, and because particles can move along the large surface of the aggregate. Nevertheless, the number of crystals and LSCs with crystals are the same (fig. 3(c)) meaning that LSCs only contain one such crystal in the initial stages of the simulation. Figures 3(b) and 3(c) imply that the crystals are small with less than ten crystalline particles.

The size of the largest clusters is not shown at long times, since it remains almost unchanged for the rest of the simulation, and the second largest cluster is on average just one particle. This points to the coexistence of the largest aggregate with gas. There are some fluctuations in the size of the first and second largest clusters indicating detachment and attachment of larger aggregates from the largest cluster. The fluctuations decay with time as a consequence of an increasing crystallinity within the system making these detachments less likely. The size of the largest cluster slowly increases. This is a kinetic phenomenon resulting from the fact that the evolution of crystallinity depends on the interface between the gas and the aggregate and from the fact that the detachment and attachment of single particles is more and more difficult, due to an increasing attractive and repulsive barrier in the denser parts of the system.

Figure 3(c) implies that LSCs can eventually contain more than one crystal. The number of crystals, and the number of LSCs with crystals, stop growing, while the number of non-crystalline LSCs starts decreasing. This indicates that no new crystals form within the LSCs, and that non-crystalline LSCs gradually dissolve at the cost 
of growing crystalline LSCs. Rearrangements at this late stage resemble coarsening rather than assembly, and happen without significantly distorting the large aggregate via single-particle or small-cluster motion. Large collective rearrangements via cluster division are less likely and rare at these later times, since they require large and unlikely energy changes. An example of such a rare reorganization is shown in fig. 3 where a branch of the disordered aggregate crystallizes, grows, detaches (time 730) and attaches (time 800) to another part of the aggregate.

We note that the local crystal order has a similar evolution both in SPMC and VMMC, despite the fact that the trajectory is different when quantified with a macroscopic observable such as the size of the largest cluster. This result is a consequence of generating the moving clusters via local energy gradients. Also, a similar number of LSCs of both kinds is observed at long times both in VMMC and in SPMC, even if one system is merged into a single aggregate and the other is not. This suggests that crystals grow from the amorphous drops, independently of whether the drops are merged or isolated. Contrary to the SPMC, we did not reach timescales where most LSCs without crystals dissolve. Figure 3(c) then shows that the number of LSCs with crystals decreases at long times, while the number of crystals remains the same. This suggests that particles in disordered parts of the cluster, forming an interface between two separate LSCs with crystals, slowly become stronger bonded particles, and eventually produce a single LSC with multiple crystalline cores.

\subsection{Coarsening in charged attractive systems}

We now examine by VMMC simulation the kinetic evolution of a low-density system with short-range attractive particles which are quenched to the same temperature as in the previous section, with the difference that the particles are charged, and repel each other weakly at long distances (SALR, see fig. 1). From the kinetic point of view, addition of a long-range repulsion to the short-range attraction decreases the depth of the attraction, thereby reducing the bond lifetime, and moving the temperature closer to the optimum temperature region for self-assembly [34]. From the thermodynamic point of view, long-range repulsion decreases the liquid-vapor critical point [47]. Our simulations are not long enough to equilibrate the system, and we do not deal with equilibrium cluster phases [30], or with small arrested nonequilibrium clusters [10]. We rather simulate large [48], metastable and spherical clusters, where particle exchange between them indicates an ongoing phase-separation, and where the fluid-solid coexistence region seems to be the equilibrium phase in the long-time limit. This system is investigated here in order to show that even a weak longrange repulsion slows down the aggregation in the presence of collective motion to an extent, where the cluster lifetime is comparable to the bond lifetime, and that clusters may (at least temporarily) recrystallize into several isolated crystalline domains.

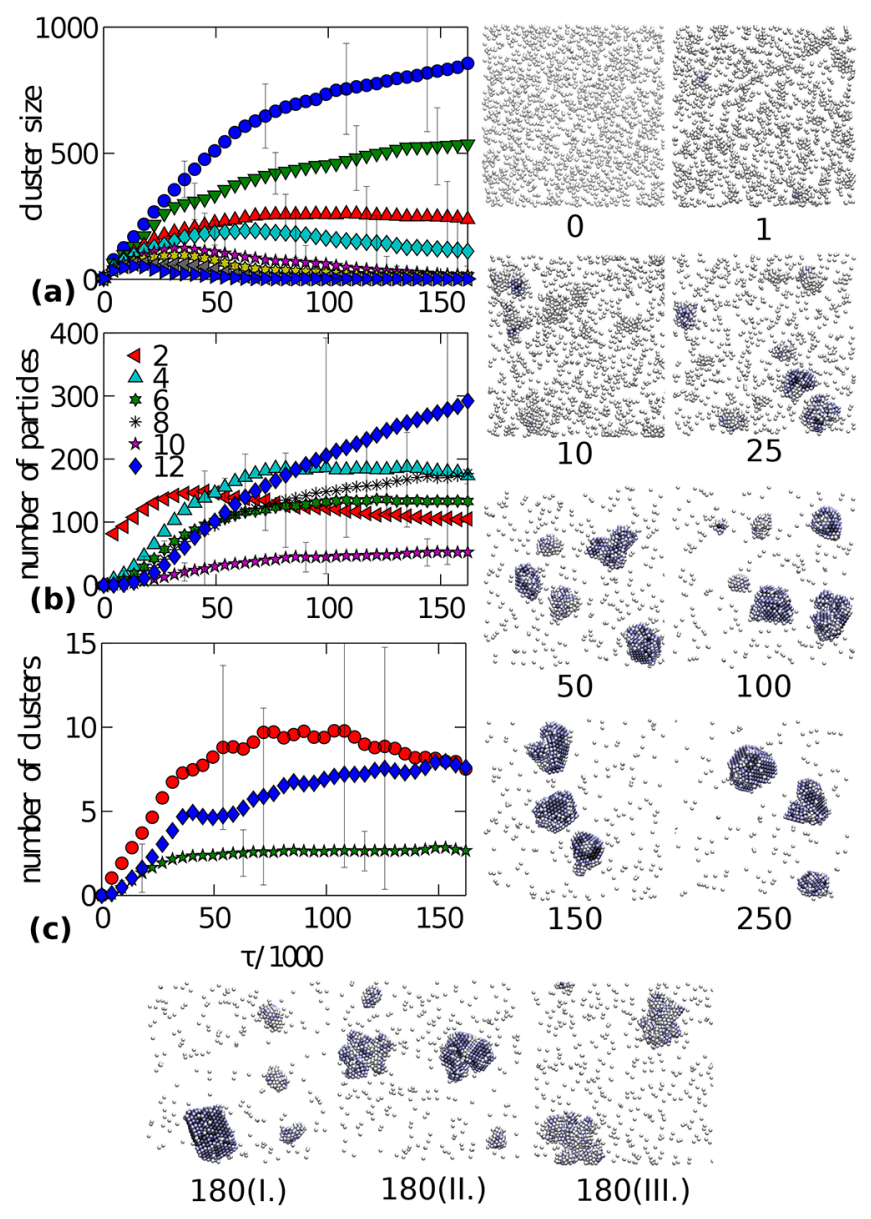

Fig. 4. (Color online) Time evolution of the system composed of particles with short-range attraction and long-range repulsion. The simulation is started from a quenched liquid state. The three figures on the bottom show possible products of the simulation at time 180 , other figures and symbols have the same meaning as in fig. 2

Figure 4 shows that after the quench, the system phase separates rapidly into several large clusters which move with respect to each other without merging into a single aggregate. Similarly to SPMC simulation of SA systems, large isolated clusters do not merge on our simulation timescale and the growth proceeds via linear regimes, which are correlated with the short- and long-scale structures. This indicates that the growth of the largest clusters in the SALR system is governed by single-particle attachment and detachment. The snapshots in fig. 4 show, indeed, that crystals grow mainly through addition of monomers to crystalline surfaces of the clusters.

The observables in fig. 4 have large fluctuations, reflecting complex energy landscapes [31,49], and kinetic trapping resulting from the finite-size effects. Indeed, the snapshots at the bottom of fig. 4 show that the simulations do not necessarily result in several separated crystals, but can also produce a single large crystalline cluster, or clusters with low internal crystal order. Figure 4 (state III.) then shows a situation where the system is trapped in a state where exchange of particles between two amorphous 
surfaces may continue for a long time. This may be due to both the delay time [5] caused by a lower nucleation rate of particles within the amorphous drops [50] or due to a finite-size kinetic effect. If the system were larger, a crystal would form with a higher probability which would then grow at the expense of amorphous structures.

\section{Scaling of the dynamics}

The probability of moving a single particle or a cluster is dependent on local forcefields in both SPMC and VMMC. This allows one to study the dynamical evolution of local crystallinity in simulations by both methods. However, similarly to the choice of the time step in a dynamical simulation, MC techniques require one to choose the size of the MC displacement. It is known that SPMC can approximate the dynamics of equilibrium fluids, if the displacement is small [51], but the suitability of MC simulation for non-equilibrium simulations such as gelation is unclear. In what follows, we discuss the role of that choice in our non-equilibrium simulation of the phase separation process.

Firstly, figs. 5(a-c) show that the properties of crystallization pathways are roughly independent of $\delta$ in SPMC simulation, if the observables are plotted as a function of $\tau$. This suggests that the SPMC simulation may realistically represent the time evolution of our non-equilibrium system, where collective motion is negligible, unimportant or may be integrated out, and that a rough timescale may be defined not only in equilibrium [51], but also in nonequilibrium MC simulations of quenched systems where phase separation and crystallization are underway.

Secondly, in figs. 5(d-f), we study the dynamics under different choices of $\delta$ in the VMMC simulation. If $\delta$ is smaller than the width of the pairwise attraction, the local forcefields are weak and only small moving clusters form. The dynamics is then similar to the SPMC. A more significant collective motion appears, only when $\delta$ is comparable to or larger than the attraction range. Large $\delta$ may, however, significantly increase the fraction of large cluster moves.

In the following, we show that the crystallization pathways in the VMMC are entirely different for different choices of $\delta$ at early times, but become independent of $\delta$ at late times. Figures 5(d) and (e) show that the cluster growth is no longer correlated with the local structure. This can be seen again from the size of the second largest cluster (fig. 5(d)), and from the concentrations of weakly bonded and crystalline particles (fig. $5(\mathrm{e})$ ), where the size reaches its maximum at different times $\tau$, and the concentrations meet at roughly the same times $\tau$. This difference is crucial for the approximation of the dynamics by the VMMC. For smaller maximum displacements $\delta$, isolated clusters move slowly, and have more time to develop a local crystal structure, before they merge with other clusters. For larger $\delta$, clusters move faster and coalesce into a disordered aggregate well before a significant local order develops. We emphasize that time $\tau$ is defined by eq. (4), which does not consider different timescales of differently
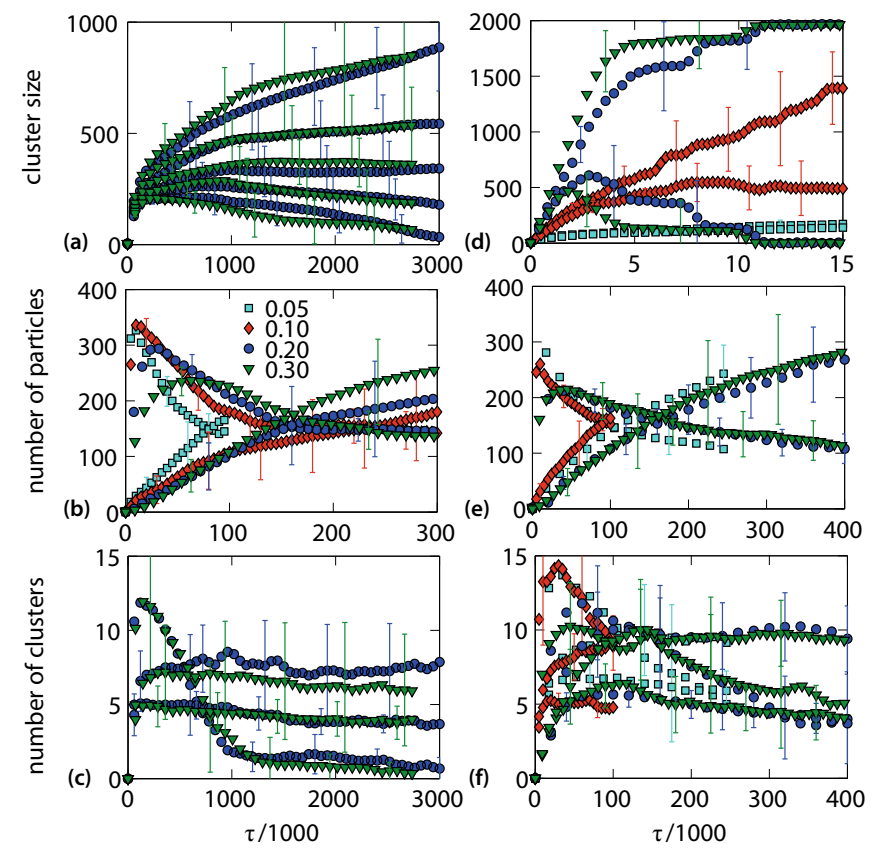

Fig. 5. (Color online) Time evolution of long- and short-range structure and its dependence on the maximum displacement size $\delta / \sigma$ in a low-density and short-range attractive system following a quench. The system is simulated using SPMC simulation (a-c) and VMMC simulation (d-f). The size of $\delta$ is in the legend of (b). Panels (a) and (d) show size of several largest clusters. (b) and (e) show the number of weakly bonded $\left(n_{b}=2\right)$ and crystalline $\left(n_{b}=12\right)$ particles. Panels (c) and (f) show the number of crystals and the number of LSCs with and without a crystal inside. The long-time data in (d) and (f) only show results for $\delta$ equal to $0.20 \sigma$ and $0.30 \sigma$, the data for shorter timescales and $\delta<0.20$ scale similarly. The averages are over 10 independent simulations.

sized clusters. The omission of this time rescaling will not affect our conclusion about different trajectories for different $\delta$, which are fixed through the course of the simulation. For example, if the time was rescaled by a constant such that the cluster sizes peak at the same time for different $\delta$, the concentrations would meet at different times, and the properties of trajectories would still be different.

One might thus expect that different choices of $\delta$ in the VMMC may lead to different kinetically slowed down states, characterized by different structural observables. However, the initial structural differences may disappear at later times of the simulation. This is shown in figs. 5(c) and (f), where observables under consideration are nearly independent of $\delta$. These results are in agreement with the previous observations that the products of the kinetically slowed down phase separation are invariant with respect to the dynamics [12]. How this scaling relates to the scaling of dynamics with the range of attraction in similar systems [52] is an interesting topic for future study.

As far as the invariance of trajectories in SALR systems is concerned, the preliminary results indicate that the properties of crystallization pathways are nearly independent of $\delta$. This is due to the fact that the phase 
separation is governed by single-particle exchange as it is in SPMC simulation of SA systems. The definition of a physical time, and the approximation of realistic dynamical pathways, is more feasible in SALR systems with several isolated long-living aggregates, than in attractive systems without long-range repulsion where a single disordered aggregate forms.

\section{Discussion}

This paper describes the MC kinetics in a low-density system after quench, and illustrates the role of collective motion when local crystallization competes with aggregation. The first part of the results shows straightforwardly qualitative relations between the crystal growth and local crystallinity in systems where collective motion is and is not allowed. The second part deserves more attention in the discussion since it shows that the choice of maximum displacement $\delta$ in our MC simulation significantly alters the ensemble of crystallization pathways. We may thus ask how to interpret the dynamics under different choices of $\delta$, and what is the optimum $\delta$ ?

The optimum size of $\delta$ depends on the aim of the VMMC simulation. If the aim is to approximate the dynamical pathways, the optimum $\delta$ can be chosen by simulating a single spherical cluster of a given size in the VMMC, and by measuring its diffusion for different choices of maximum $\delta$. The optimum $\delta$ is the one which matches most closely the diffusion coefficient given analytically by the Stokes equation for the spherical cluster [41]. However, several of these tests should be done for differently sized clusters and a different optimal $\delta$ should be expected from each of those tests. The size of average $\delta$ which best matches the diffusion coefficients of differently sized clusters is then calculated in a further optimization. These tests [41] involve an approximation which assumes that the cluster is spherical, crystalline, and has permanent bonds, which guarantee that the cluster does not break during the test. The present paper shows for a simple system that these assumptions do not hold, during the simulation of gelation or kinetically slowed down phase separation where clusters are not spherical, are allowed to break, have low internal crystallinity, and their acceptance probability may change during the course of the phase separation (see also SI). Although the diffusion test on crystalline clusters [41] is probably the simplest approach to choose the optimum $\delta$, there may still be alternative approaches to vary $\delta$ during the course of the simulation as is discussed below. We emphasize that despite all the approximations, the VMMC is believed [41] to simulate the dynamics of phase separation more realistically than BD, which does not distinguish between diffusion coefficients of differently sized clusters. The approaches discussed below should further improve the simulation.

Another aim of the VMMC simulation may be to capture a physically realistic product of kinetically slowed down phase separation or to reach the equilibrium state of the system in a minimum amount of computational time. This is a sensible question since our results indicate that the structure of the kinetically slowed down aggregate is invariant with respect to the choice of $\delta$, and the same naturally applies for the equilibrium state. The maximum displacement and the overall crystallinity vs CPU time is then a suitable measure of efficiency. Our preliminary results show that larger values of $\delta$ become ineffective in local equilibration of the system at later stages of the VMMC simulation, as most of the time is spent on moving large clusters, and that a small value of $\delta(\delta \leq 0.05)$ eventually becomes the most efficient.

Hierarchical self-assembly. A slow variation of $\delta$ during the course of the simulation may result in a multi-scale modeling protocol, which efficiently captures the collective motion on a scale which is characteristic of a specific time window of the self-assembly. In hierarchical selforganization [48], this scale increases with time, and the size of $\delta$ could, for example, be made to depend on the average size of crystals in the system. The early stages of this protocol would be associated with the shortest $\delta$, the shortest physical timescales, and with the self-assembly of the smallest building units; the later stages by a larger $\delta$, longer times, and self-assembly at a scale given by the size of the aggregates self-assembled earlier. Under the assumption that local motion is important on one scale, but not important later at a larger scale, local motion at late stages would be made less frequent, but would not be completely forbidden. Another assumption is that slow variation of $\delta$ violates the detailed balance condition for the sampling to an extent which is negligible compared to the effects of coarse-graining and the non-equilibrium nature of the quench.

We can use our SA system to speculate about a protocol varying $\delta$ during the simulation such that the aggregation happens via the motion of isolated crystals. The VMMC simulation of the quench would start with a sufficiently low $\delta$. Our results indicate that the system phase separates into a large number of clusters and that the first crystals appear. As implied by our VMMC with $\delta$, or by using Brownian Dynamics [8], the clusters remain isolated for the timescale of the simulation and the aggregates may crystallize. The system then develops a transient but long enough time window, where the crystals can be temporally described on another scale [9], as new and larger renormalized spherical particles with attractions that are shorter ranged relative to the size of the drops. Moreover, the attractive potential of the drops may be stronger than that between the constituent particles, because a contact between the drops can be formed by more than one contact between the constituent particles. This leads to a deeper well or equivalently to a larger $\epsilon$ in the renormalized system, with the position of the state point in the phase diagram being changed in two ways. The relatively shorterranged attraction moves the fluid-solid binodal and the critical point to lower temperatures [3]. Since $\epsilon$ and $T$ are equivalent through $k_{\mathrm{B}} T / \epsilon$, the deeper well has a similar effect, and moves the state point to a lower part of the phase diagram. The latter effect is stronger, because a single aggregate of drops is formed rather than a fluid of drops in our simulations. In hierarchical self-assembly [48], it is desirable to avoid dynamic arrest at the scale of the drops, 
and to produce spherical clusters of drops rather than a disordered cluster from drops. It is possible that there is a simulation protocol, where $T$ and $\delta$ are varied slowly and simultaneously, producing large long-living spherical aggregates from the smaller scale renormalized spheres. This protocol may or may not correspond to physical reality.

Approaches to vary $\delta$. The maximum MC displacement $\delta$ may not only represent a parameter of the simulation coarsening the dynamics, but may also be related to a dissipative quantity such as viscosity. The above protocol for realistic dynamics or efficient sampling may be achieved in different ways. Instead of drawing $\delta$ from a uniform distribution, $\delta$ drawn from another, possibly Gaussian, distribution, may result in a more realistic distribution of moving clusters approximating the dynamics. One could also draw an analogy between the optimum size of the particle displacement in the VMMC and the optimum pseudo-temperature regulating the linking strength in static cluster algorithms [53,54]. A pseudo-temperature in the VMMC algorithm can be introduced by using another temperature in the linking function, and correcting with a bias in the acceptance probability. This approach is equivalent to using fictitious potentials for linking, and real potentials for accepting, the moving clusters [23]. This pseudo-temperature may then be slowly changed instead of $\delta$. Rapid periodic changes of $\delta$ are also possible in SPMC simulations, but can bring the system out of equilibrium sampling [55]. Changes of $\delta$ or the pseudo-temperature in the MC simulations during assembly may also be stepwise and follow a real-time feedback. A systematic change of bond strength can, indeed, improve crystal yield [56]. How $\delta$ and its changes are represented in experiments requires more attention, but microwave heating [57] can be used to locally disturb the bonds, the time control of bond strength is achievable by exposure to light in repeated pulses [58], and hierarchical self-assembly in DNA origami can be achieved by a slow cooling process [59].

Very low density and Brownian dynamics. Perez et al. [8] performed Brownian dynamics (BD) simulations at very low packing fractions $\phi=0.001$, using the same potentials as in eq. (1) with no long-range repulsion $(A=0)$. Their system was quenched to temperatures $T=0.10$ and $T=0.20$, producing isolated spherical aggregates with well developed crystallinity. The aggregates did not merge into a single cluster on the timescale of their long BD simulations. To compare with these results, and to investigate the role of collective MC motion for a larger $\delta$, we performed VMMC simulations on the same system. We used $\delta=0.20 \sigma$ and the Brownian scaling of proposed clusters according to step 2(b) of the algorithm. The result of the VMMC simulation was a system which rapidly merged into one large fractal-like aggregate, without having enough time to develop spherical clusters with a high internal crystallinity. The difference between BD and VMMC for large $\delta$ is consistent with the results of this paper, suggesting that for large $\delta$, clusters aggregate quickly with respect to local crystallization. Whether it is $\mathrm{BD}$ or VMMC that approximates the dynamics in a more realistic way may require a comparison with experiment.

\section{Conclusion}

By using MC simulations, we have characterized the products of medium quenches in SA systems, and we analyzed the corresponding crystallization pathways. In accordance with what has been reported before $[3,8]$, the quench is followed by condensation into liquid drops which remain isolated for a long time, and aggregation of which can not be easily studied by conventional dynamical simulations. Our MC methods allow us to tackle this timescale to show that, if the dominant mechanism for structural evolution is the single-particle motion, and contribution from the collective modes of motion can be neglected or integrated out, the condensation and growth of the drops follows three linear growth regimes correlated with local order. The first regime is the formation of drops with and without a crystal inside; the second regime is a dissolution of amorphous structures with a low internal crystal order; and the third regime is a slow growth of crystals via adsorption of single particles from dissolving usually smaller crystals. The optimum conditions for this situation seem to be intermediately strong quenches of systems at low densities to temperatures corresponding to the intersection of the spinodal and the attractive glass line [15, 13], and lying within the optimum temperature range for self-assembly [56]. The collective motion is unimportant in systems where long-range repulsion inhibits the aggregation. In systems where collective motion affects the dynamics, the correlated linear growth is not observed, and local crystallization is preceded by larger scale aggregation. The crystals then grow at the expense of the amorphous parts of the aggregate.

The collective dynamics for a fixed $\delta$ studied in this paper is not necessarily physically realistic, but it is believed to be more accurate than other simulation approaches, such as Brownian dynamics, where different diffusion constants of differently sized clusters are not explicitly dependent on the cluster size and geometry. The sensitivity of crystallization pathways to different choices of $\delta$ may be significant, and is examined in the second part of the paper. The pathways are on average independent of the choice of $\delta$ in the SPMC simulation, but differ in the VMMC simulation of the attractive system especially for large $\delta$. If $\delta$ is small, aggregates do not move, and the VMMC simulation is similar to the SPMC simulation with local crystallinity of isolated droplets being developed first. If $\delta$ is large, the system aggregates into liquid clusters which quickly merge into a single, large, and disordered aggregate, with local crystallinity being developed afterwards. The structural features of the aggregates, however, seem to become independent of $\delta$ at later times, suggesting that the kinetically slowed down structures observed in this paper have a universal significance and may be physical.

The results provide evidence that the dynamical Monte Carlo methods, studied here, and used elsewhere $[41,45$, 60-62], predict realistic products of phase separation, despite the fact that different choices of $\delta$ may lead to different initial crystallization pathways. The methods are of potential use in modeling the dynamics or in finding 
the products of phase separation in those systems where collective modes of motion contribute to overall dynamics [18], where anisotropic interactions govern the crystallization pathways [41], where hydrodynamics affects the onset of gelation, and where alternative dynamical methods $[63-65,40]$ are not sufficient to capture the important physical phenomena behind the self-assembly.

We thank G. Foffi, M. Sweatman, A. Troisi, B. Vorselaars for comments, and D. Quigley for the code analyzing local crystallinity. This work was supported by the Engineering and Physical Sciences Research Council (grant number EP/I001514/1). This Programme Grant funds the Materials Interface with Biology (MIB) consortium. We also acknowledge support from the Warwick Postgraduate Research Scholarship scheme, and we thank the Centre for Scientific Computing of the University of Warwick for computational resources.

Open Access This is an open access article distributed under the terms of the Creative Commons Attribution License (http://creativecommons.org/licenses/by/4.0), which permits unrestricted use, distribution, and reproduction in any medium, provided the original work is properly cited.

\section{References}

1. E.R. Leite, C. Ribeiro, Crystallization and Growth of Colloidal Nanocrystals (Springer, Berlin, 2012).

2. H. Cölfen, M. Antonietti, Mesocrystals and Nonclassical Crystallization (Wiley, 2008).

3. G.A. Vliegenthart, J.F.M. Lodge, H.N.W. Lekkerkerker, Phys. A 263, 378 (1999).

4. P.R. ten Wolde, D. Frenkel, Science 277, 1975 (1997).

5. D. Kashchiev, P.G. Vekilov, A.B. Kolomeisky, J. Comput. Phys. 122, 244706 (2005).

6. A.F. Wallace, L.O. Hedges, A. Fernandez-Martinez, P. Raiteri, J.D. Gale, G.A. Waychunas, S. Whitelam, J.F. Banfield, J.J. De Yoreo, Science 341, 885 (2013).

7. P.J. Lu, J.C. Conrad, H.M. Wyss, A.B. Schofield, D.A. Weitz, Phys. Rev. Lett. 96, 28306 (2006).

8. T. Perez, Y. Liu, W. Li, J.D. Gunton, A. Chakrabarti, Langmuir 27, 11401 (2011).

9. M.E. Cates, M. Fuchs, K. Kroy, W.C.K. Poon, A.M. Puertas, J. Phys.: Condens. Matter 16, S4861 (2004).

10. E. Mani, W. Lechner, W.K. Kegel, P.G. Bolhuis, Soft Matter 10, 4479 (2014).

11. A. Fortini, E. Sanz, M. Dijkstra, Phys. Rev. E 78, 041402 (2008).

12. G. Foffi, C. De Michele, F. Sciortino, P. Tartaglia, J. Chem. Phys. 122, 224903 (2005).

13. S. Sastry, Phys. Rev. Lett. 85, 590 (2000).

14. V. Testard, L. Berthier, W. Kob, J. Chem. Phys. 140, 165402 (2014).

15. E. Zaccarelli, J. Phys.: Condens. Matter 19, 323101 (2007).

16. P.J. Lu, E. Zaccarelli, F. Ciulla, A.B. Schofield, F. Sciortino, D.A. Weitz, Nature 453, 499 (2008).

17. Y. Xia, T.D. Nguyen, M. Yang, B. Lee, A. Santos, P. Podsiadlo, Z. Tang, S.C. Glotzer, N.A. Kotov, Nat. Nanotechnol. 6, 580 (2011).
18. S. Whitelam, E.H. Feng, M.F. Hagan, P.L. Geissler, Soft Matter 5, 1251 (2009).

19. P. Charbonneau, D.R. Reichman, Phys. Rev. E 75, 011507 (2007).

20. S. Whitelam, P.L. Geissler, J. Chem. Phys. 127, 154101 (2007).

21. F. Sciortino, P. Tartaglia, E. Zaccarelli, J. Phys. Chem. B 109, $21942(2005)$

22. D. Frenkel, Proc. Natl. Acad. Sci. U.S.A. 101, 17571 (2004).

23. S. Whitelam, Mol. Simulat. 37, 606 (2011).

24. B. Chen, J.I. Siepmann, J. Phys. Chem. B 104, 8725 (2000).

25. J.W. Liu, E. Luijten, Phys. Rev. Lett. 92, 035504 (2004).

26. S. Růžička, M.P. Allen, Phys. Rev. E 89, 033307 (2014).

27. Š. Růžička, M.P. Allen, Phys. Rev. E 90, 033302 (2014).

28. C.N. Likos, H. Löwen, M. Watzlawek, B. Abbas, O. Jucknischke, J. Allgaier, D. Richter, Phys. Rev. Lett. 80, 4450 (1998).

29. A.A. Louis, E. Allahyarov, H. Löwen, R. Roth, Phys. Rev. E 65, 061407 (2002).

30. F. Sciortino, S. Mossa, E. Zaccarelli, P. Tartaglia, Phys. Rev. Lett. 93, 055701 (2004).

31. S. Mossa, F. Sciortino, P. Tartaglia, E. Zaccarelli, Langmuir 20, 10756 (2004).

32. A. de Candia, E. Del Gado, A. Fierro, N. Sator, M. Tarzia, A. Coniglio, Phys. Rev. E 74, 010403 (2006).

33. S.J. Khan, C.M. Sorensen, A. Chakrabarti, J. Chem. Phys. 131, 194908 (2009).

34. D. Klotsa, R.L. Jack, Soft Matter 7, 6294 (2011).

35. J. Grant, R.L. Jack, Phys. Rev. E 85, 021112 (2012).

36. V. Testard, L. Berthier, W. Kob, Phys. Rev. Lett. 106, 125702 (2011).

37. J.C.F. Toledano, F. Sciortino, E. Zaccarelli, Soft Matter 5, 2390 (2009).

38. C.P. Royall, A. Malins, Faraday Discuss. 158, 301 (2012).

39. M.P. Allen, D.J. Tildesley, Computer simulation of liquids (Oxford University Press, 1987).

40. M.P. Allen, D. Quigley, Mol. Phys. 111, 3442 (2013).

41. T.K. Haxton, S. Whitelam, Soft Matter 8, 3558 (2012).

42. T. Schilling, H.J. Schöpe, M. Oettel, G. Opletal, I. Snook, Phys. Rev. Lett. 105, 25701 (2010).

43. P.J. Steinhardt, D.R. Nelson, M. Ronchetti, Phys. Rev. B 28, 784 (1983)

44. P.R. ten Wolde, M.J. Ruiz-Montero, D. Frenkel, J. Chem. Phys. 104, 9932 (1996).

45. J. Grant, R.L. Jack, S. Whitelam, J. Chem. Phys. 135, 214505 (2011).

46. E. Zaccarelli, W.C.K. Poon, Proc. Natl. Acad. Sci. U.S.A. 106, 15203 (2009).

47. D. Pini, G. Jialin, A. Parola, L. Reatto, Chem. Phys. Lett. 327, 209 (2000).

48. M.B. Sweatman, R. Fartaria, L. Lue, J. Chem. Phys. 140, 124508 (2014).

49. A.J. Archer, N.B. Wilding, Phys. Rev. E 76, 031501 (2007).

50. Š. Růžička, Ph.D. thesis, University of Warwick (2014).

51. E. Sanz, D. Marenduzzo, J. Chem. Phys. 132, 194102 (2010).

52. G. Foffi, C. Michele, F. Sciortino, P. Tartaglia, Phys. Rev. Lett. 94, 78301 (2005).

53. A. Troisi, V. Wong, M.A. Ratner, J. Chem. Phys. 122, $024102(2005)$. 
54. A. Bhattacharyay, A. Troisi, Chem. Phys. Lett. 458, 210 (2008).

55. R.H. Swendsen, Phys. Procedia 15, 81 (2011).

56. D. Klotsa, R.L. Jack, J. Chem. Phys. 138, 094502 (2013).

57. M. Godinho, C. Ribeiro, E. Longo, E.R. Leite, Cryst. Growth Des. 8, 384 (2008).

58. P.K. Jha, V. Kuzovkov, B.A. Grzybowski, M.O. de la Cruz, Soft Matter 8, 227 (2012).

59. P.W.K. Rothemund, Nature 440, 297 (2006).

60. J. Russo, P. Tartaglia, F. Sciortino, Soft Matter 6, 4229 (2010)
61. N.S. Bieler, T.P.J. Knowles, D. Frenkel, R. Vácha, PLoS Comput. Biol. 8, e1002692 (2012).

62. D. Fusco, P. Charbonneau, Phys. Rev. E 88, 012721 (2013).

63. S. Plimpton, J. Comput. Phys. 117, 1 (1995), http://lammps.sandia.gov/index.html.

64. J.M. Ilnytskyi, M.R. Wilson, Comp. Phys. Commun. 134, 23 (2001).

65. J.A. Anderson, C.D. Lorenz, A. Travesset, J. Comput. Phys. 227, 5342 (2008), ISSN 0021-9991, http://www. sciencedirect.com/science/article/pii/ S0021999108000818. 